\title{
Probing the breakdown of topological protection: Filling-factor-dependent evolution of robust quantum Hall incompressible phases
}

\author{
T. Tomimatsu $\odot,{ }^{1}$ K. Hashimoto $\odot,{ }^{1,2, *}$ S. Taninaka, ${ }^{1}$ S. Nomura $\odot,{ }^{3}$ and Y. Hirayama ${ }^{1,2,4}$ \\ ${ }^{1}$ Graduate School of Sciences, Tohoku University, Sendai 980-8578, Japan \\ ${ }^{2}$ Centre for Spintronics Research Network, Tohoku University, Sendai 980-8578, Japan \\ ${ }^{3}$ Division of Physics, University of Tsukuba, Tennodai, Tsukuba 305-8571, Japan \\ ${ }^{4}$ Center for Science and Innovation in Spintronics (Core Research Cluster), Tohoku University, Sendai 980-8577, Japan
}

(Received 17 December 2018; revised manuscript received 25 November 2019; accepted 2 December 2019; published 5 February 2020)

\begin{abstract}
The integer quantum Hall $(\mathrm{QH})$ effects characterized by topologically quantized and nondissipative transport are caused by an electrically insulating incompressible phase that prevents backscattering between chiral metallic channels. We probed the incompressible area susceptible to the breakdown of topological protection using a scanning-gate technique incorporating nonequilibrium transport. The obtained pattern revealed the filling-factor$(v-)$ dependent evolution of the microscopic incompressible structures located along the edge and in the bulk region. We found that these specific structures, respectively, attributed to the incompressible edge strip and bulk localization, show good agreement in terms of $v$-dependent evolution with a calculation of the equilibrium $\mathrm{QH}$ incompressible phases, indicating the robustness of the $\mathrm{QH}$ incompressible phases under the nonequilibrium condition. Further, we found that the $v$ dependency of the incompressible patterns is, in turn, destroyed by a large imposed current during the deep QH effect breakdown. These results demonstrate the ability of our method to image the microscopic transport properties of a topological two-dimensional system.
\end{abstract}

DOI: 10.1103/PhysRevResearch.2.013128

A two-dimensional electron system (2DES) subjected to strong magnetic fields forms a quantum Hall $(\mathrm{QH})$ insulating phase with a state lying in a gap between quantized Landau levels (LLs). This gapped phase, the so-called incompressible phase, prevents backscattering between the metallic gapless (compressible) phase counterpropagating along both sides of the 2DES edges [1]. This is the key microscopic aspect of nondissipative chiral transport of the integer QH effect, which is characterized by a longitudinal resistance that vanishes and a universal quantized Hall conductance protected by a topological invariant $[2,3]$. Topological phases are attracting renewed attention due to the recent discovery of exotic topological materials such as insulators [4-7], superconductors [8], and Weyl semimetals [9].

The formation of incompressible and compressible phases in the $\mathrm{QH}$ regime originates from the interplay between Landau quantization and the Coulomb interaction [10], which drives nonlinear screening $[11,12]$. The spatial configuration depends on the potential landscape. For example, the edge confinement potential, accompanied by strong bending of the LLs, forms spatially alternating unscreening and screening regions due to the Fermi-level pinning at the gap and LLs. These regions, respectively, result in alternating incompressible and compressible strips near the 2DES edge. The in-

\footnotetext{
*hashi@tohoku.ac.jp

Published by the American Physical Society under the terms of the Creative Commons Attribution 4.0 International license. Further distribution of this work must maintain attribution to the author(s) and the published article's title, journal citation, and DOI.
}

nermost incompressible strip moves and spreads to the bulk as the LL filling factor $v$ reduces to an integer $i$ from a higher $v$. This $v$ dependency of the edge strips has been microscopically investigated using various imaging techniques such as single-electron transistor imaging [13], Hall-potential imaging [14,15], microwave impedance imaging [16], capacitance imaging [17]. Additionally, scanning probe study on edge transport has been performed with scanning-gate imaging [18-24] for various systems, and it has been extended with superconducting quantum interference device (SQUID) magnetometry for a topological spin-Hall insulator [25].

In the bulk incompressible region formed at $v \simeq i$, the disorder potential plays an important role in giving rise to isolated compressible puddles that result in $\mathrm{QH}$ localization [26-28]. QH localized states have been probed [29,30], and they were demonstrated to undergo phase transition to a delocalized state with a scanning tunneling microscope [31], which accounts for the transition from nondissipative to dissipative transport.

By contrast, for a practical sample such as a Hall bar, microscopic pictures of the $\mathrm{QH}$ effect have not been fully understood [32]. For instance, the contribution of the innermost incompressible strip to nondissipative transport is debatable [33]. Moreover, the microscopic mechanism of the breakdown of topological protection is thought to originate from backscattering through the incompressible region [34,35], and this has recently become a key issue in research on the quantum spin Hall effect [23,36] and anomalous QH effect [37]. To understand the transport properties inherent to the $\mathrm{QH}$ effect, it is important to elucidate the microscopic aspects of $\mathrm{QH}$ transport in real devices, namely, how the innermost incompressible region evolves in the Hall bar and, hence, 


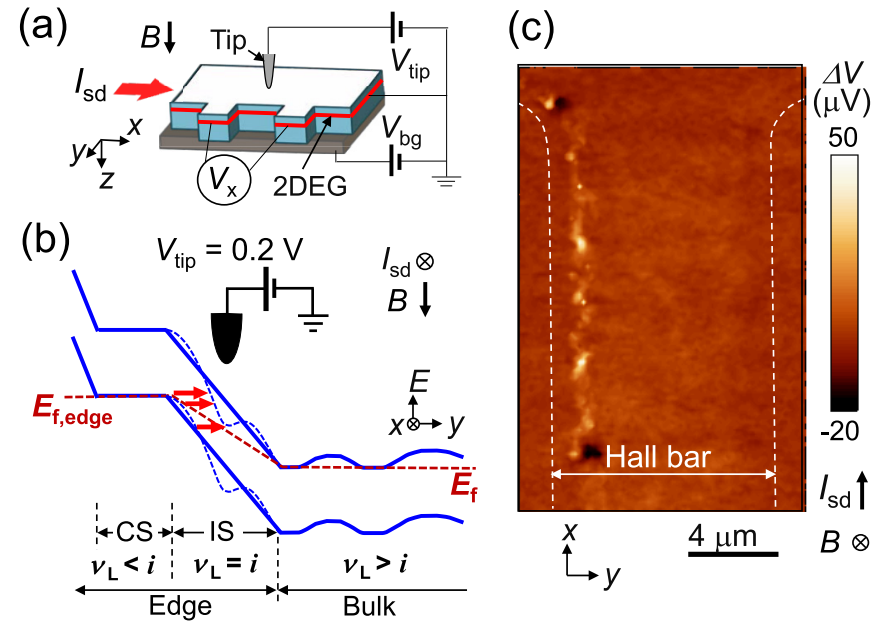

FIG. 1. (a) Schematic of the experimental setup of the SGM. $V_{\mathrm{x}}$ is recorded as the tip is scanned at $V_{\text {tip }}$ with $I_{\text {sd }}$ and $B$, respectively, applied in the $x$ and $z$ directions. (b) Schematic of inter-LL tunneling (marked by red arrows) between two LL subbands near the edge of the higher $\mu_{\text {chem }}$ side under the nonequilibrium condition, which is derived from the deviation between the Fermi energies in the edge $\left(E_{\mathrm{f}, \text { edge }}\right)$ and bulk $\left(E_{\mathrm{f}}\right)$ compressible regions. Tip-induced LL bending, indicated by the blue dashed line, enhances inter-LL tunneling. The incompressible and compressible strips are indicated by "IS" and "CS," respectively. "Edge" and "bulk" indicate the edge strips and 2DES bulk region, respectively. The magnitudes of $v_{\mathrm{L}}$ with respect to the exact integer $i$ are also shown for each region. (c) SGM image of a tip-induced $V_{\mathrm{x}}$ change $(\Delta V)$ at $v=2.27, I_{\mathrm{sd}}=3.1 \mu \mathrm{A}$, and $B=4 \mathrm{~T}$; dashed lines denote the Hall bar edges. The line noise was removed using 2D Fourier filtering.

limits nondissipative, topologically quantized transport. Here, we present the ability of a scanning-gate method incorporating a nonequilibrium transport technique to pinpoint the areas susceptible to breakdown of topological protection and hence gives access to the local breakdown at a nonequilibrium transport. We found the robust $\mathrm{QH}$ nature, i.e., the evolution of the innermost incompressible $\mathrm{QH}$ phase from the edge strip to bulk localization, and the microscopic aspects of the global $\mathrm{QH}$ breakdown, namely, the breakdown of the $\mathrm{QH}$ effect, under a larger nonequilibrium condition.

To probe the local breakdown of the incompressible area, we used a powerful tool [the scanning-gate microscope (SGM)], which uses resistive detection as transport measurements and hence directly images transport channels with a high sensitivity at a high spatial resolution [38]. In topological phases such as QH [18-21,39], and quantum spin Hall [23], the SGM has been widely used to probe an important local transport characteristic, namely, backscattering between counterpropagating edge channels. To capture a sufficiently intense signal from backscattering, one that is strongly suppressed at $v \simeq i$, a conventional SGM requires the electrostatic influence of a large negative tip voltage $V_{\text {tip }} \leqslant-1 \mathrm{~V}$ [20,21,39], moving the incompressible strip across a narrow channel. However, the tip-induced displacement of the incompressible strip may locally disturb the intrinsic structure.

In our setup shown in Fig. 1(a), to minimize global perturbation, the tip voltage was set to $V_{\text {tip }} \sim 0.2 \mathrm{~V}$, corresponding to the value of the contact potential mismatch between the tip and the sample [40]. To address the $\mathrm{QH}$ phase at $v \simeq i$ and obtain a local signal without applying a large tip voltage, we incorporated nonequilibrium transport. We investigated a 2DES that was confined in a 20 -nm-wide $\mathrm{GaAs} / \mathrm{Al}_{0.3} \mathrm{Ga}_{0.7} \mathrm{As}$ quantum well located $165 \mathrm{~nm}$ beneath the surface. The wafer was processed into a $10-\mu \mathrm{m}$-wide Hall bar. The mobility of the 2DES was $\mu=130 \mathrm{~m}^{2} \mathrm{~V}^{-1} \mathrm{~s}^{-1}$ at an electron density $n=1.8 \times 10^{15} \mathrm{~m}^{-2}$. Figure 1 (b) depicts the alternating compressible and incompressible regions formed along an edge of the Hall bar. The local $v\left(v_{\mathrm{L}}\right)$ of an incompressible strip is maintained at $v_{\mathrm{L}}=i$, while the bulk $v$ is modified by sweeping $B$ or $n_{\mathrm{s}}$ tuned by a back gate voltage $\left(V_{\mathrm{bg}}\right)$. To achieve the nonequilibrium condition, we increased the source-drain current $\left(I_{\mathrm{sd}}\right)$ until the Hall voltage deviated from the $\mathrm{QH}$ condition. The imposed Hall voltage predominantly enhances the potential slope within the innermost incompressible region [15], inducing inter-LL tunneling from the edge to the bulk through the innermost incompressible strip. Then, an electron is backscattered into the opposite edge channel through compressible or directional-hopping channels along the Hall electric field. This leads to a dissipative current $[41,42]$, and thus a nonzero longitudinal resistance. The tip locally provides small electric perturbation to the 2DES owing to the effective potential mismatch rearranged by imposed excess Hall voltage, and eventually bends the LL locally and, hence, increases the inter-LL tunneling rate, as shown in Fig. 1(b) (details are discussed in the Supplemental Material [40]). The tip-induced inter-LL tunneling further enhances backscattering and hence the longitudinal voltage $\left(V_{\mathrm{x}}\right)$. By mapping the resulting $\Delta V$, we can visualize the innermost incompressible region. All measurements were performed at a sample temperature below $250 \mathrm{mK}$.

Figure 1(c) shows a typical SGM image obtained by capturing $\Delta V$ at $v=2.27(B=4 \mathrm{~T})$ under the nonequilibrium conditions at $I_{\mathrm{sd}}=3.1 \mu \mathrm{A}$. A distinct linelike pattern can be seen extending in the $x$ direction along a Hall bar edge (left dashed line), which corresponds to the side with the higher chemical potential $\left(\mu_{\text {chem }}\right)$ across the $y$ direction of the Hall bar. This $\mu_{\text {chem }}$ dependency, confirmed by reversing the direction of the current [40], can be explained by the fact that $\mu_{\text {chem }}$ mainly drops at the higher- $\mu_{\text {chem }}$ incompressible strip in a nonequilibrium condition [42], where we expect a higher rate of inter-LL scattering [43] and thus more SGM sensitivity with respect to the corresponding incompressible strip. To minimize the influence of $I_{\mathrm{sd}}$ on the incompressible patterns, $I_{\text {sd }}$ was limited to below the current in all measurements, at which the position of the strip shows no significant $I_{\text {sd }}$ dependence in the entire measurement region of $v$. Otherwise, there is a deviation associated with global $\mathrm{QH}$ breakdown, as discussed in the Supplemental Material [40].

We examined the $v$ dependence of the SGM patterns. The measurements were performed at $I_{\mathrm{sd}}$, which was tuned to maintain a constant offset $V_{\mathrm{x}}\left(V_{\mathrm{x}} \sim 1 \mathrm{mV}\right)$ at each $v$ (for details regarding the conditions, see the Supplemental Material [40]). Figure 2(a) displays representative SGM images captured near $v=2$ and tuned with the gate controlled $n_{\mathrm{s}}$ at constant $B(B=4 \mathrm{~T})$. Decreasing $v$ from 2.17 , the position of the line pattern shifts and widens to the bulk of 2DES. The same tendency of the $\nu$-dependent patterns was also observed in the same area near $v=4$ at $B=2 \mathrm{~T}$, as shown in Fig. 2(b). 
(a)

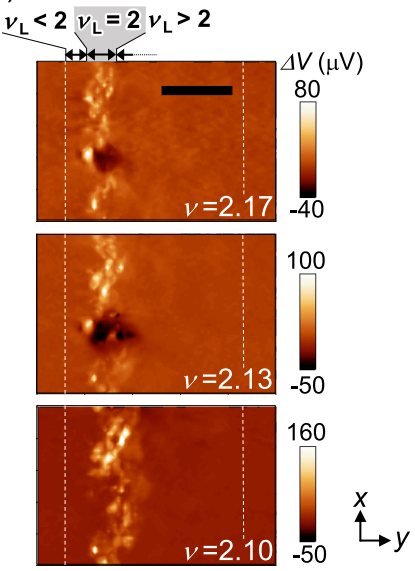

(c)
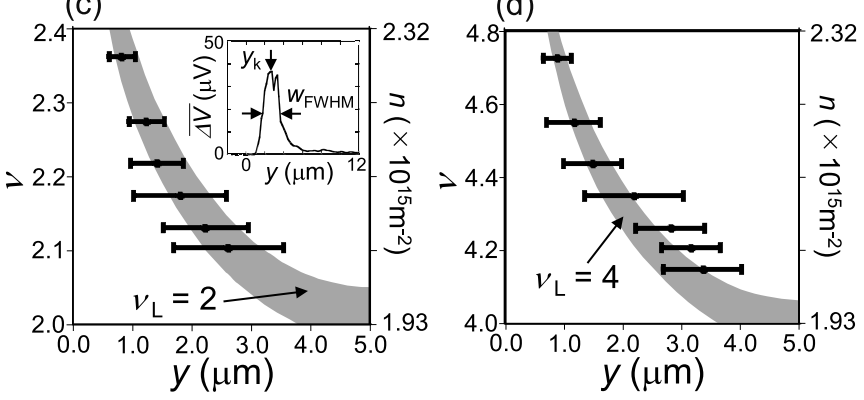

FIG. 2. SGM measurements near $v=2$ and 4. (a), (b) Representative $\Delta V$ images at different $v$ tuned by $n$ at (a) $B=4$ T near $v=2$ and (b) $B=2 \mathrm{~T}$ near $v=4$ (see the Supplemental Material [40] for the corresponding longitudinal and Hall resistances at equilibrium in the $\mathrm{QH}$ regime). The scale bar is $4 \mu \mathrm{m}$. Dashed lines denote the Hall bar edges. The line noise was removed using 2D Fourier filtering. As $v$ decreases toward integer $i$, the patterns are further enhanced, such that the full scale of contrast is appropriately optimized for clarity. (c), (d) Position (dot) and width (bar) of the $\Delta V$ peak as a function of $v$ near (c) $v=2$ and (d) 4 . The position and width are, respectively, determined by the distance from the Hall bar edge $\left(y_{\mathrm{k}}\right)$ and the full width at half-maximum $\left(W_{\mathrm{FWHM}}\right)$. These are extracted from the cross-sectional $\Delta V$ profile spatially averaged over the $x$ region $(\overline{\Delta V})$, as indicated in the inset in (c) [obtained from the image for $v=2.10$ in (a)]. Here, $v=2.0-2.4$ and $4.0-4.8$ are selected to ensure the same $n_{\mathrm{s}}$ range $1.93 \times 10^{15}-2.32 \times 10^{15} \mathrm{~m}^{-2}$. The gray area denotes the incompressible regions determined by LSDA calculation for (c) $\nu_{\mathrm{L}}=2$ and (d) 4 .

We extracted the positions $\left(y_{\mathrm{k}}\right)$ and width $\left(W_{\mathrm{FWHM}}\right)$ of the line patterns, which were respectively defined as the first moment (for details, see the Supplemental Material [40]) and the full width at half-maximum in a $\Delta V$ profile after spatially averaging over the $8.5-\mu \mathrm{m}$ range in the $x$ direction $(\overline{\Delta V})$, as shown in the inset of Fig. 2(c). For a quantitative comparison of the observed $\Delta V$ peak positions, we performed a calculation in the Landau gauge based on the local spin-density approximation (LSDA) $[44,45]$ using a typical potential profile [46] in the QH regime $\left(I_{\mathrm{sd}}=0 \mathrm{~A}\right)$ (for details regarding the calculations, see the Supplemental Material [40]). The vdependent incompressible region, experimentally determined from $y_{\mathrm{k}}$ (dots) and $W_{\mathrm{FWHM}}$ (bars), is compared with the innermost $\mathrm{QH}$ incompressible region (gray area) calculated by the LSDA for $v_{\mathrm{L}}=2$ in Fig. 2(c) and $v_{\mathrm{L}}=4$ in Fig. 2(d). (a)
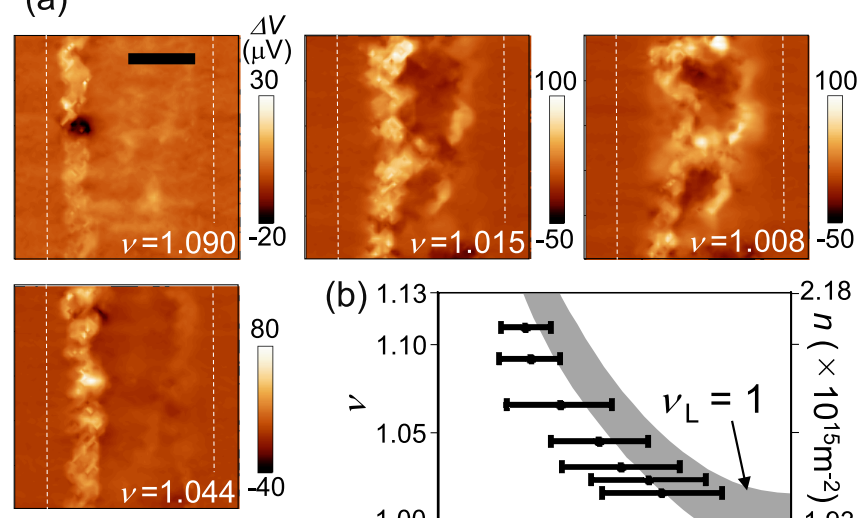

(b) 1.13
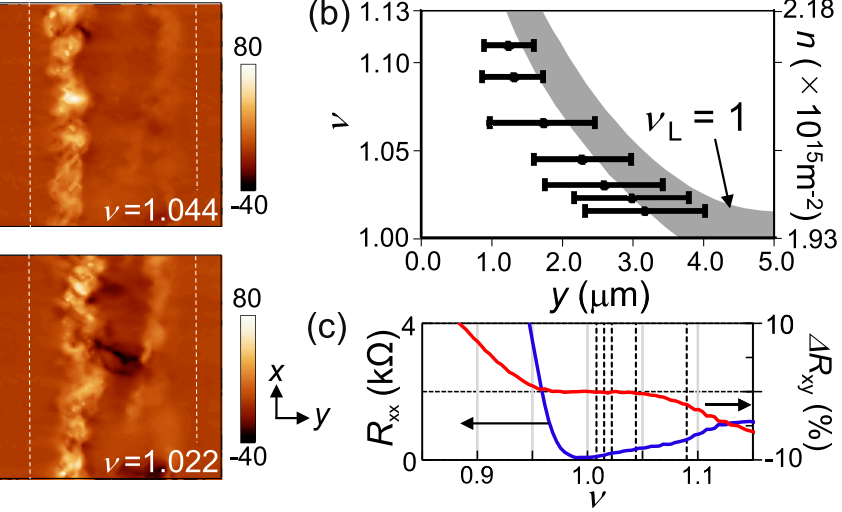

FIG. 3. SGM measurements near $v=1$. (a) Representative $\Delta V$ images taken at different $v$ tuned by $n$ at $B=8 \mathrm{~T}$. The scale bar is $4 \mu \mathrm{m}$. Dashed lines denote the Hall bar edges. The line noise was removed by 2D Fourier filtering. (b) Positions $y_{\mathrm{k}}$ (dots) and widths $W_{\text {FWHM }}$ (bars) of the $\overline{\Delta V}$ profile as a function of $v$. Gray area: the calculated incompressible region for $v_{\mathrm{L}}=1$. (c) Longitudinal $\left(R_{\mathrm{xx}}\right)$ and normalized Hall $\left(\Delta R_{\mathrm{xy}}\right)$ resistance curves measured for the same Hall bar at the equilibrium $\mathrm{QH}$ condition, $I_{\mathrm{sd}}=10 \mathrm{nA}$, at $B=8 \mathrm{~T}$. Vertical dashed lines mark the $v$ positions at which the SGM images shown in (a) were captured.

We found good agreement between the experimental results and the LSDA calculation for both values of $\nu$. Additionally, a closer examination of the line pattern shows local fluctuation in the same region at both values of $v$, e.g., in the bottom half of the images taken at $v=2.17$ in Fig. 2(a) and at $v=4.43$ in Fig. 2(b). This implies that the edge incompressible strip meanders along the equipotential line disturbed by potential disorder.

The same technique was further applied to the spin-gap incompressible region emerging at odd $v_{\mathrm{L}}$. Figure 3(a) displays SGM images captured near $v=1$ at $B=8 \mathrm{~T}$. The $v_{\mathrm{L}}=1$ incompressible strip was observed as a straight line that moves from the higher- $\mu_{\text {chem }}$ edge (at $v=1.090$ ) to the interior of the Hall bar (at $v=1.022$ ). As seen in Fig. 3(b), the measured $y_{\mathrm{k}}$ shifts, and $W_{\mathrm{FWHM}}$ widens with decreasing $v$, which is again consistent with the incompressible region (gray area) evaluated with the LSDA calculations that considered the exchange enhancement of the spin gap.

To examine the incompressible bulk localization, we focused on the $v$ region closer to $v=i($ here, $v=1)$ in which the incompressible region is expected to extend over the entire bulk region, as expected by the LSDA calculation and as shown in the gray region in Fig. 3(b). At $v=1.015$ and 1.008 [Fig. 3(a)], we found a closed-loop pattern in the incompressible region. Notably, this $v$ region corresponds to that in which the global longitudinal and Hall resistances at equilibrium in the $\mathrm{QH}$ regime [Fig. 3(c)] exhibit a dip and plateau, respectively. The same tendency was also observed 
(a)

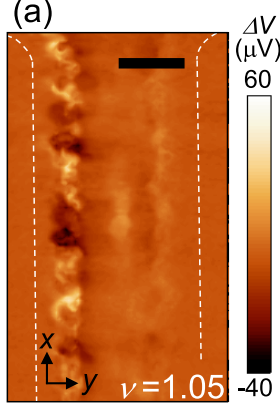

(d)
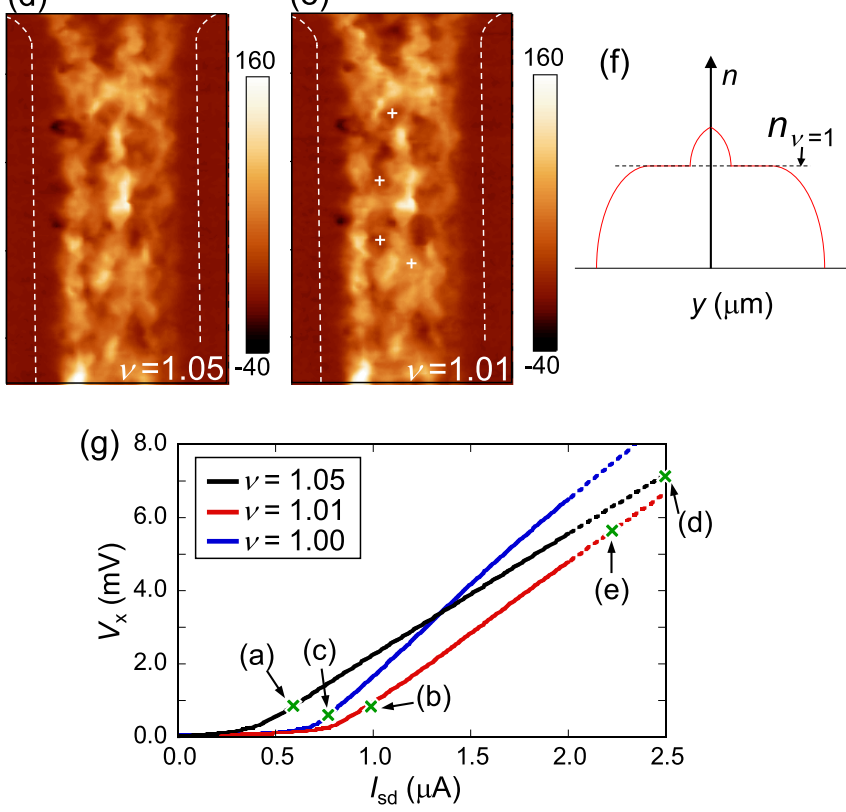

FIG. 4. Filling factor and electrical current evolution of SGM patterns taken near $v=1$ at $B=6 \mathrm{~T}$ (see the Supplemental Material [40] for corresponding $R_{\mathrm{xx}}$ and $\Delta R_{\mathrm{xy}}$ at equilibrium in the $\mathrm{QH}$ regime). (a)-(c) Filling-factor-dependent structures of the $v_{\mathrm{L}}=1$ incompressible phase taken at low $I_{\mathrm{sd}}(0.6-1.0 \mu \mathrm{A})$ for $v=1.05$, 1.01 , and 1.00 . (d), (e) SGM patterns obtained at high $I_{\text {sd }}(2.2-$ $2.5 \mu \mathrm{A}$ ) for $v=1.05$ and 1.01 . The scale bar is $4 \mu \mathrm{m}$; dashed lines denote the Hall bar edges. The line noise was removed using 2D Fourier filtering. (f) Schematic density profile along a red line across a closed-loop pattern in (c). (g) $V_{\mathrm{x}}-I_{\mathrm{sd}}$ curves for $v=1.05,1.01$, and 1.00. Crosses mark the measurement conditions for (a)-(e).

in a wider spatial region at different $B$, namely, $B=6 \mathrm{~T}$, as shown in Figs. 4(a)-4(c). In particular, Fig. 4(c) shows distinct closed-loop patterns (around white crosses) over the entire Hall bar in the $x$ direction. The observed loop structure is attributed to an incompressible barrier encircling compressible puddles [29] where electrons accumulate to screen the potential valley, as depicted in Fig. 4(f) and discussed in Supplemental Material [40]. The average distance between the structures was estimated to be about $3 \mu \mathrm{m}$, which is of the same order as the separation of the potential-disorder-related states (a few $\mu \mathrm{m}$ ) observed in a similar modulation-doped quantum well [47].

The observed $v$ evolution of the incompressible phases that shows agreement with the LSDA calculation indicates that the $\mathrm{QH}$ phases are microscopically robust against the local breakdown caused by inter-LL scattering under the nonequilibrium condition. The robustness of a $\mathrm{QH}$ state is supported by our previously reported scanning nuclear resonance imaging [43], where we demonstrated the spatial homogeneity of the fully polarized $v=1$ state maintained under similar nonequilibrium conditions near the onset of breakdown of the $\mathrm{QH}$ effect. We speculate that this robustness maintains at nonequilibrium $I_{\mathrm{sd}}$ up to the limit above which the position of the incompressible pattern showed the $I_{\text {sd }}$ dependence (see the Supplemental Material [40]).

To explore how $\mathrm{QH}$ systems are microscopically broken by a larger imposed current, we examined the current-induced evolution of the patterns by imposing $I_{\mathrm{sd}}$ up to 2-5 times higher than those used for the low-current images [Figs. 4(a)-4(c)], as indicated by the $V_{\mathrm{x}}-I_{\mathrm{sd}}$ curves [Fig. 4(g)]. As shown in the Supplemental Movies [40], both the local incompressible patterns near the edge [Fig. 4(a)] and in the bulk region [Fig. 4(b)] gradually expand with increasing $I_{\mathrm{sd}}$ to the compressible region, eventually covering the entire region and exhibiting a dense filament pattern independent of $v$ [Figs. 4(d)-4(e)]. The observed vanishing of the $v$ dependency in the patterns clearly indicates a global breakdown of the v-dependent $\mathrm{QH}$ effect.

Compared with the $\mathrm{QH}$ incompressible pattern enclosing the compressible puddle [Fig. 4(c)], the observed filament pattern shows a wider distribution and corrugates at a shorter length scale. Notably, the observed filaments partially surround the positions marked by crosses [Fig. 4(e)], which correspond to the positions [crosses in Figs 4(c)] of the disorder-induced $\mathrm{QH}$ compressible puddles. These indicate that the filament pattern is correlated with the potential disorder whose potential slope may not be fully screened owing to less compressibility induced by the heating effect $[48,49]$ in the dissipative $\mathrm{QH}$ breakdown regime $[43,50]$. This implies that inter-LL scattering arises along the potential disorder [51] over the sample in the deep QH breakdown regime.

In conclusion, using a nonequilibrium-transport-assisted SGM technique, we demonstrated the robustness of the microscopic structures of the incompressible $\mathrm{QH}$ phases contributing to topologically quantized and nondissipative transport in a Hall bar. In the deep QH breakdown regime, the observed $v$-dependent characteristics vanish and are unified into a disorder-related pattern, suggesting that microscopic breakdown arises along the potential disorder of the sample. In our future research, we shall use this powerful method to attain a microscopic understanding of nondissipative transport, in the fractional QH effect and other topological edgetransport effects of topological insulators. Our method can probe local properties of topological protection, e.g., by imaging the backscattering sites from the helical edge channel to electron puddles [52]. As such, it can tackle topical issues, such as the suppression of the quantized conductance of the quantum spin Hall effect [23,36] and the nonzero longitudinal resistance of the anomalous quantum Hall effect [37,53], which can be caused by backscattering. Moreover, our method can be applied to research on the hydrodynamics of the $\mathrm{QH}$ fluid $[35,54]$ and extended to the hydrodynamics of Dirac fluid [55]. 
We thank K. Muraki and NTT for supplying high-quality wafers, K. Sato and K. Nagase for sample preparation, M. H. Fauzi for helpful discussion, and Y. Takahashi for figure preparation. K.H. and T.T. acknowledge the JSPS for financial support: KAKENHI Grants No. 17H02728 and No.
18K04874, respectively. Y.H. acknowledges support from the JSPS (KAKENHI Grants No. 15H05867, No. 15K21727, and No. 18H01811), and S.N. also acknowledges the JSPS for their support (Grant No. 15H03673). K.H. and Y.H. thank Tohoku University's GP-Spin program for support.
[1] T. Chakraborty and P. Pietiläinen, The Quantum Hall effects: Integral and Fractional, Vol. 85 (Springer, Berlin, 2013).

[2] D. J. Thouless, M. Kohmoto, M. P. Nightingale, and M. denNijs, Phys. Rev. Lett. 49, 405 (1982).

[3] Y. Hatsugai, Phys. Rev. Lett. 71, 3697 (1993).

[4] C. L. Kane and E. J. Mele, Phys. Rev. Lett. 95, 146802 (2005).

[5] C. L. Kane and E. J. Mele, Phys. Rev. Lett. 95, 226801 (2005).

[6] B. Bernevig, T. Hughes, and S.-C. Zhang, Science 314, 1757 (2006)

[7] C. Z. Chang, J. Zhang, X. Feng, J. Shen, Z. Zhang, M. Guo, K. Li, Y. Ou, P. Wei, L. L. Wang, Z. Q. Ji, Y. Feng, S. Ji, X. Chen, J. Jia, X. Dai, Z. Fang, S. C. Zhang, K. He, Y. Wang et al., Science 340, 167 (2013).

[8] L. Fu and C. L. Kane, Phys. Rev. Lett. 100, 096407 (2008).

[9] A. A. Burkov and L. Balents, Phys. Rev. Lett. 107, 127205 (2011).

[10] D. B. Chklovskii, B. I. Shklovskii, and L. I. Glazman, Phys. Rev. B 46, 4026 (1992).

[11] U. Wulf, V. Gudmundsson, and R. R. Gerhardts, Phys. Rev. B 38, 4218 (1988).

[12] A. L. Efros, F. G. Pikus, and V. G. Burnett, Phys. Rev. B 47, 2233 (1993).

[13] A. Yacoby, H. F. Hess, T. A. Fulton, L. N. Pfeiffer, and K. W. West, Solid State Commun. 111, 1 (1999).

[14] E. Ahlswede, P. Weitz, J. Weis, K. Von Klitzing, and K. Eberl, Phys. B (Amsterdam) 298, 562 (2001).

[15] J. Weis and K. V. Klitzing, Philos. Trans. R. Soc. London A 369, 3954 (2011).

[16] K. Lai, W. Kundhikanjana, M. A. Kelly, Z.-X. Shen, J. Shabani, and M. Shayegan, Phys. Rev. Lett. 107, 176809 (2011).

[17] M. Suddards, A. Baumgartner, M. Henini, and C. J. Mellor, New J. Phys. 14, 083015 (2012).

[18] M. T. Woodside, C. Vale, P. L. McEuen, C. Kadow, K. D. Maranowski, and A. C. Gossard, Phys. Rev. B 64, 041310(R) (2001).

[19] A. Baumgartner, T. Ihn, K. Ensslin, K. Maranowski, and A. C. Gossard, Phys. Rev. B 76, 085316 (2007).

[20] N. Paradiso, S. Heun, S. Roddaro, L. Sorba, F. Beltram, G. Biasiol, L. N. Pfeiffer, and K. W. West, Phys. Rev. Lett. 108, 246801 (2012).

[21] N. Pascher, C. Rössler, T. Ihn, K. Ensslin, C. Reichl, and W. Wegscheider, Phys. Rev. X 4, 011014 (2014).

[22] M. R. Connolly, R. K. Puddy, D. Logoteta, P. Marconcini, M. Roy, J. P. Griffiths, G. A. C. Jones, P. A. Maksym, M. Macucci, and C. G. Smith, Nano Lett. 12, 5448 (2012).

[23] M. König, M. Baenninger, A. G. F. Garcia, N. Harjee, B. L. Pruitt, C. Ames, P. Leubner, C. Brüne, H. Buhmann, L. W. Molenkamp, and D. Goldhaber-Gordon, Phys. Rev. X 3, 021003 (2013).

[24] Z. Dou, S. Morikawa, A. Cresti, S. W. Wang, C. G. Smith, C. Melios, O. Kazakova, K. Watanabe, T. Taniguchi, S.
Masubuchi, T. Machida, and M. R. Connolly, Nano Lett. 18, 2530 (2018).

[25] K. C. Nowack, E. M. Spanton, M. Baenninger, M. König, J. R. Kirtley, B. Kalisky, C. Ames, P. Leubner, C. Brüne, H. Buhmann, L. W. Molenkamp, D. Goldhaber-Gordon, and K. A. Moler, Nat. Mater. 12, 787 (2013).

[26] B. Huckestein, Rev. Mod. Phys. 67, 357 (1995).

[27] A. Struck and B. Kramer, Phys. Rev. Lett. 97, 106801 (2006).

[28] C. Sohrmann and R. A. Römer, New J. Phys. 9, 97 (2007).

[29] S. Ilani, J. Martin, E. Teitelbaum, J. Smet, D. Mahalu, V. Umansky, and A. Yacoby, Nature (London) 427, 328 (2004).

[30] G. A. Steele, R. C. Ashoori, L. N. Pfeiffer, and K. W. West, Phys. Rev. Lett. 95, 136804 (2005).

[31] K. Hashimoto, C. Sohrmann, J. Wiebe, T. Inaoka, F. Meier, Y. Hirayama, R. A. Römer, R. Wiesendanger, and M. Morgenstern, Phys. Rev. Lett. 101, 256802 (2008).

[32] K. von Klitzing, Phys. Rev. Lett. 122, 200001 (2019).

[33] A. Siddiki and R. R. Gerhardts, Phys. Rev. B 70, 195335 (2004).

[34] L. Eaves, P. S. S. Guimaraes, J. C. Portal, T. P. Pearsall, and G. Hill, Phys. Rev. Lett. 53, 608 (1984).

[35] L. Eaves, Phys. B (Amsterdam) 256, 47 (1998).

[36] S. Wu, V. Fatemi, â. Quinn, D. Gibson, K. Watanabe, T. Taniguchi, R. J. Cava, and P. Jarillo-Herrero, Science 359, 76 (2018).

[37] Y. Xing, F. Xu, Q. F. Sun, J. Wang, and Y. G. Yao, J. Phys.: Condens. Matter 30, 435303 (2018).

[38] M. Topinka, B. J. LeRoy, S. Shaw, E. Heller, R. Westervelt, K. Maranowski, and A. Gossard, Science 289, 2323 (2000).

[39] N. Aoki, C. R. da Cunha, R. Akis, D. K. Ferry, and Y. Ochiai, Phys. Rev. B 72, 155327 (2005).

[40] See Supplemental Material at http://link.aps.org/supplemental/ 10.1103/PhysRevResearch.2.013128 for details on the methods of the SGM measurement, experimental analysis, and LSDA calculation, and for movies showing $I_{\mathrm{sd}}$ evolution of the incompressible patterns.

[41] L. Eaves and F. W. Sheard, Semicond. Sci. Technol. 1, 346 (1986).

[42] K. Panos, R. Gerhardts, J. Weis, and K. von Klitzing, New J. Phys. 16, 113071 (2014).

[43] K. Hashimoto, T. Tomimatsu, K. Sato, and Y. Hirayama, Nat. Commun. 9, 2215 (2018).

[44] S. Nomura and Y. Aoyagi, Phys. Rev. Lett. 93, 096803 (2004).

[45] S. Mamyouda, H. Ito, Y. Shibata, S. Kashiwaya, M. Yamaguchi, T. Akazaki, H. Tamura, Y. Ootuka, and S. Nomura, Nano Lett. 15, 2417 (2015).

[46] K. Güven and R. R. Gerhardts, Phys. Rev. B 67, 115327 (2003).

[47] J. Hayakawa, K. Muraki, and G. Yusa, Nat. Nanotechnol. 8, 31 (2013). 
[48] T. Machida, H. Hirai, S. Komiyama, T. Osada, and Y. Shiraki, Phys. Rev. B 54, R14261(R) (1996).

[49] M. Kato, A. Endo, S. Katsumoto, and Y. Iye, Phys. Rev. Lett. 102, 086802 (2009).

[50] S. Komiyama, Y. Kawaguchi, T. Osada, and Y. Shiraki, Phys. Rev. Lett. 77, 558 (1996).

[51] K. Güven, R. R. Gerhardts, I. I. Kaya, B. E. Sağol, and G. Nachtwei, Phys. Rev. B 65, 155316 (2002).
[52] J. I. Väyrynen, M. Goldstein, and L. I. Glazman, Phys. Rev. Lett. 110, 216402 (2013).

[53] M. Kawamura, R. Yoshimi, A. Tsukazaki, K. S. Takahashi, M. Kawasaki, and Y. Tokura, Phys. Rev. Lett. 119, 016803 (2017).

[54] A. M. Martin, K. A. Benedict, F. W. Sheard, and L. Eaves, Phys. Rev. Lett. 91, 126803 (2003).

[55] M. Mendoza, H. J. Herrmann, and S. Succi, Phys. Rev. Lett. 106, 156601 (2011). 